\title{
Neighborhood Upper and Lower Approximation for the Content- Based Image Retrieval
}

\author{
Zhao Jie ${ }^{1,2}$ and Xie Gang ${ }^{1, *}$ \\ ${ }^{1}$ College of Information Engineering, Taiyuan University of Technology, Shanxi, \\ China \\ ${ }^{2}$ Department of Computer Engineering, Taiyuan College, Shanxi, China \\ E-mail:tydxcomputer@163.com,xiegang@tyut.edu.cn
}

\begin{abstract}
In order to solve the problem that the dimension disaster caused by high-dimensional features seriously affects the efficiency of the content-based image retrieval, this paper presents a method for generating an image retrieval algorithm with neighborhood rough set. By introducing the upper and lower approximation definition of neighborhood rough set, the proposed algorithm achieves the features selection and similarity measurement for automatic image retrieval. Experiment shows that the proposed algorithm is effective in comparison with the other mentioned methods.
\end{abstract}

Keywords: Image retrieval; Neighborhood; Upper approximation; Lower approximation

\section{Introduction}

In the current age of digitalization, images are one of the most important media sources from which people can acquire information. Due to more intuitive capabilities of information presentation, each day numerous digital images are produced and transmitted over the network. Furthermore, making images easily retrievable has been a challenge since very large image collection that is becoming more and more common. Therefore, developing a quick and accurate digital image retrieval system to deal with the tremendous amount of digital images has become a very important research topic in which many scholars. Researchers are very interested in the content-based image retrieval (CBIR), where image contents are described by the features such as color, texture and shape. The more the dimension of features are, the better features describes the image contents. But the dimension disaster caused by high-dimensional features seriously affects the efficiency of the image retrieval. Therefore, as the preprocessing step of image retrieval, the feature selection not only to ensure the effectiveness of features, but also to maintain the reduction of features. As a result, how to select the features relates to the effectiveness and efficiency of the image retrieval [1-2].

Rough set theory forms a vehicle to deal with the uncertain information and inexact knowledge, and the nature implied in the data is able to be found with systemic mathematical approaches. The theory has been widely used in artificial intelligence, pattern recognition, data mining, system analysis and other fields. Neighborhood rough set extends application of the rough set to support numeric attributes, which is more suitable for the selection of image features. In order to balance the effectiveness of image features and the reduction of image features, we proposed a method of high-dimension image features selection based on neighborhood rough set. By the concept of the neighborhood, the method can find the subsets of the images with the similar features and confirm the dependency of the features that is the basic measure of attribution reduction.

* Corresponding author. Professor of Discipline of Control Science \& Engineering 
At the same time, the similarity of the images is quantified according to the upper and lower approximations.

The rest of this paper is organized as follow. We first briefly overview the related works in Section 2. Section 3 introduces the proposed approach and its theory basic. Section 4 contains experimental results obtained by applying our algorithms and other algorithms to image retrieval. Finally Section 5 is the conclusion of the work and future prospects.

\section{Related Works}

Feature selection is referred to as selecting subset of attributes from the set of original attributes. The purpose of the feature selection is to identify the significant features, eliminate the irrelevant of dispensable features and build a good learning model [3]. Nowadays numerous successful implementations of feature selection and classification of various applications using rough set are available. Rough set theory has been introduced by Zdzislaw Pawlak to deal with imprecise or vague concepts in the early 1980s [4]. Rough set can handle the ambiguous and imperfect data by taking in the ideas of "limited set" and "equivalent relation" of set theory, and newly introducing the concepts of "classification" and "approximation". In recent years we witnessed a rapid growth of interest in rough set theory and its applications, worldwide. Researchers focused their attention on reduction and classification algorithms based on rough set [5-7].

Khoo et al. [8] proposed a novel approach for the rule induction of inconsistent information systems. It was achieved by integrating rough set with a statistics-based inductive learning algorithm. Chen et al. [9] presented a reasonable definition of parameterization reduction of soft sets and compared it with the attribute reduction in rough set. By using this new definition of parameterization reduction, they improved the application of a soft set in a decision-making problem. Thangavel et al. [10] designed Modified Quickreduct algorithm for horizontal and vertical reduction. At the same time, they discussed the performance study of various reduction algorithms such as Quickreduct and Variable Precision Rough Set (VPRS) for constructing the efficient rules. Questier et $a l$. [12] introduced the rough set theory to construct reductions in a supervised way for reducing the number of features in an unsupervised clustering.

However, the equivalence relations are directly induced from nominal attributes based on the attribute value so that the rough set model cannot support numeric attributes in real-world applications. Y.Y. Yao [12] proposed a binary relation based framework for the study of neighborhood systems and rough set approximations. Within this framework, these two notions may be formulated, interpreted, and compared. He studied the class of 1-neighborhood systems in which each element has one neighborhood. Hu et al. [13] generalized two rough set models, named $\delta$ neighborhood rough set and $k$-nearestneighbor rough set, for mixed numerical and categorical feature selection and reduction. A forward greedy mixed attribute reduction algorithm is constructed to find minimal subsets of features which can keep classification ability based on the proposed model. Y. $\mathrm{Yu}$ [14] introduced neighborhood rough set model into multi-label classification for automatic image annotation. They consider the uncertainty of the mapping from visual feature space to semantic concepts space. Experiments show that the proposed method achieves more promising performance in comparison with to some well-known multilabel learning algorithms. 


\section{The Proposed Features Selection and Similarity Measurement Algorithm}

The paper introduces neighborhood rough set into image retrieval, including features selection and similarity quantization. In the following, we define several important concepts of neighborhood rough set, which are the theoretical basis of the proposed algorithm. Then, the optimal features are selected by utilizing the defined concepts of neighborhood rough set in order to complete the reduction process of sample attributes. At finally, the similarity measurement of the images according to the reduced features is explained in detail.

\subsection{Neighborhood Rough Set}

Formally, let universe $I S=<U, A, V, f>$ be the samples for image retrieval, where $U$ is the finite and nonempty set of samples $\left\{x_{1}, x_{2}, \ldots, x_{n}\right\}$ and $x_{i}(i=1, \ldots, n)$ is arbitrary image of the image database, $n$ is the number of the images in the database. $A$ denotes the nonempty set of attributes $\left\{a_{1}, a_{2}, \ldots, a_{K}\right\}$, i.e. the categories of the image features, and $K$ is the number of the categories of the image features. Because the classification of the images is unknown before image retrieval, $A$ is a set of condition attributions, and is not a decision attribute. $V$ denotes the set of the values of the features, which is $\left\{a_{i}^{1}, \ldots, a_{1}^{m_{1}}\right\} \bigcup\left\{a_{2}^{1}, \ldots, a_{2}^{m_{2}}\right\} \ldots \bigcup\left\{a_{k}^{1}, \ldots, a_{k}^{m_{k}}\right\}$ and $m_{k}$ is the dimension of the image features $a_{k}$. The map function $\mathrm{f}$ shows the relationship $f: U \times A \rightarrow V$.

Neighborhood of $x_{i}$ is a subset of samples close to $x_{i}$. In the application of the image retrieval, $x_{i}$ denotes an image, and neighborhood of $x_{i}$ is a subset of the images which features close to $x_{i}$. Whatever, the first issue is to give a metric to compute the difference of the features between the images.

Definition 1. A metric $\Delta$ is the distance function which describes the difference of the features between the image $x_{i}$ and $x_{j}$ :

$$
\Delta\left(x_{i}, x_{j}\right)=\left(\sum_{k=1}^{K}\left|V\left(x_{i}, a_{k}\right)-V\left(x_{j}, a_{k}\right)\right|^{p}\right)^{1 / p}
$$

Where $V\left(x_{i}, a_{k}\right)$ denotes the value of feature $a_{k}$ of the image $x_{i}$, and $V\left(x_{j}, a_{k}\right)$ denotes the value of feature $a_{k}$ of the image $x_{j}$.

The metric satisfies the following properties:

Property 1: $\forall x_{1}, x_{2} \in U, \Delta\left(x_{1}, x_{2}\right) \geq 0$; if and only if, $\Delta\left(x_{1}, x_{2}\right)=0$.

Property 2: $\forall x_{1}, x_{2} \in U, \Delta\left(x_{1}, x_{2}\right)=\Delta\left(x_{2}, x_{1}\right)$

Property 3: $\forall x_{1}, x_{2}, x_{3} \in U, \Delta\left(x_{1}, x_{3}\right) \leq \Delta\left(x_{1}, x_{2}\right)+\Delta\left(x_{2}, x_{3}\right)$

Given a nonempty set $X$ and a metric function $\Delta$, we say $X$ is a metric space, denoted by $\langle X, \Delta\rangle$. It is easy to show that $\Delta$ in the formula (1) satisfies the properties of a general metric function. With the presented metric function, we define the neighborhood of the images.

Definition 2. Given an information table $I S=\langle U, A, V, f\rangle$ and the metric $\Delta$ to describe the images, then the neighborhood $\delta$ of arbitrary image $x_{i}$ is defined as

$$
\delta_{A}\left(x_{i}\right)=\left\{x_{j} \mid x_{j} \in U, \Delta\left(x_{i}, x_{j}\right) \leq \delta, \delta \geq 0\right\}
$$

$\delta_{A}\left(x_{i}\right)$ is a subset of the images close to the image $x_{i}$ according to the features $A$, so $\delta_{A}\left(x_{i}\right)$ can also be called a neighborhood information granule induced by feature $A$ and 
image $x_{i}$. The family of neighborhood information granules $\left\{\delta_{A}\left(x_{i}\right) \mid x_{i} \in U\right\}$ forms a set of elemental concepts in the universe to approximate arbitrary subset of the samples space.

Definition 3. Given the neighborhood information granules $\delta_{A}\left(x_{i}\right)$ induced by the feature $A$ and the image $x_{i}$, and an arbitrary subset $X$ of the samples set $U$, then with respect to neighborhood relation $N_{A}$ the lower and upper approximation of $X$ is defined as

$$
\begin{array}{r}
\underline{N_{A}} X=\left\{x_{i} \mid \delta\left(x_{i}\right) \subseteq X, x_{i} \in U\right\} \\
\overline{N_{A}} X=\left\{x_{i} \mid \delta\left(x_{i}\right) \cap X \neq \varnothing, x_{i} \in U\right\}
\end{array}
$$

Suppose $B$ is the subset of the features set $A$, the neighborhood concepts have the following properties:

Property 4: If $B_{1} \subseteq B_{2} \subseteq A$, then $\forall x_{i} \in U, \delta_{B_{2}}\left(x_{i}\right) \subseteq \delta_{B_{1}}\left(x_{i}\right)$.

Property 5: If $B_{1} \subseteq B_{2} \subseteq A, X \subseteq U$ and $\delta_{B_{1}}(x) \subseteq \underline{B_{1} X}$, then $\delta_{B_{2}}(x) \subseteq \underline{B_{2}} X$.

Property 6: If $B_{1} \subseteq B_{2} \subseteq A, X \subseteq U$, then $\underline{B_{1}} X \subseteq \underline{B_{2}} X$.

\subsection{Feature Selection Algorithm}

The image database is considered as the samples set and the image features are regarded as the attributes of the samples, we adopt the definitions and properties of neighborhood rough set to confirm approximation subset of each image. On that basis, the most effective features are selected. While the essence of feature selection is to complete the reduction process of sample attributes.

In order to establish the measure standard of attribute reduction, the dependence of the image features is defined according to neighborhood rough set theory.

Definition 4. Let $B \subseteq A$ that is to say $B$ is the subset of the features set $A . X$ denotes a subset of the samples set $U . \overline{N_{B}} X, \underline{N_{B} X}$ are neighborhood upper and lower approximations of $X$ based on $B$, respectively. The sample set $U$ is the entire image database. Then the dependency degree of the image features $B$ is defined as

$$
r(B)=\frac{\operatorname{Card}\left(N_{B} X\right)}{\operatorname{Card}(U)}
$$

where $\operatorname{Card}(\square)$ is the cardinal number and denotes the number of the elements in the set.

Features selection is a process of finding an optimal subset of features from the original features set. In Definition 4, the dependency degree function reflects the capability of features $B$ to describe the image. If the dependency degree of features $A$ equals the dependency degree of features B, i.e. $r(A)=r(B)$. Meanwhile, removing arbitrary element will depress the dependency degree of features $B$, i.e. $r(B-b)<r(B), \forall b \in B$. Obviously, $B$ is the minimal subset of features $A$, which has the same describing capability as $A$. So $B$ is the optimal subset of features $A$.

According to Definition 4, the complete description of features selection is given in Algorithm 1. 


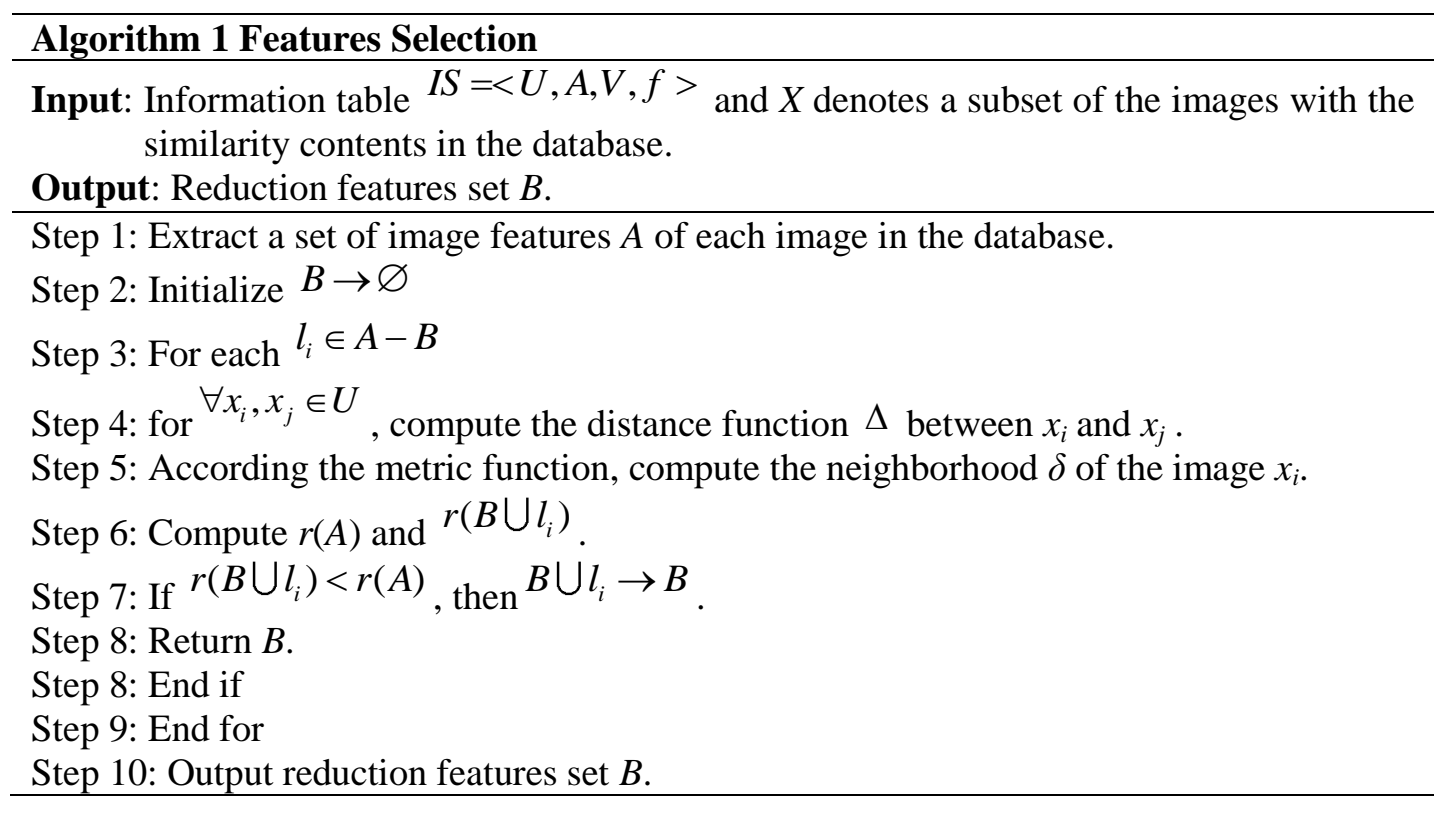

\subsection{Approach for Computing Image Similarity}

In CBIR, a very common technique is to use standard image databases with sets of different topics such as the Corel collection. When performing the image retrieval, users input a query image and find the most similar images by comparing with the images in the database. In fact, the similarity ratio of the images is measured by utilizing the similar relationship of the features between the query image and the sampled image in the database. According to algorithm 1, the optimal features have been selected from the extracted original features set. So how to quantify the similarity ratio of the images corresponding to the selected features becomes the key question of the image retrieval. The distance metric is the most frequently used quantization method [15-17]. The method is easy to be calculated and realized. However, the distance metric is regarded as the unique standard of similarity measurement so that it does not consider the uncertainty of the features in the similarity measurement.

Neighborhood rough set theory extends the conventional similarity measurement in order to handle the ambiguous and imperfect information. By introducing the lower approximation and upper approximation of neighborhood rough set, our method obtains high predictive performance compared to a variety of complex methods while maintaining low computational complexity. The similarity ratio between the query image and the sampled image can be expressed through the following definition.

Definition 5. Suppose $Q$ denotes the query image, $S_{i}$ the arbitrary sampled image in the database, then the similarity ratio between $Q$ and $S_{i}$ is defined as

$$
\begin{aligned}
& \operatorname{Sim}\left(S_{i}, Q\right)=\alpha \underline{\operatorname{Sim}}\left(S_{i}, Q\right)+(1-\alpha) \overline{\operatorname{Sim}}\left(S_{i}, Q\right) \\
& \overline{\operatorname{Sim}}\left(S_{i}, Q\right)=\frac{\left|\overline{N_{B}}\left(X_{Q}\right) \cap \overline{N_{B}}\left(X_{S_{i}}\right)\right|}{\left|\overline{N_{B}}\left(X_{Q}\right) \bigcup \overline{N_{B}}\left(X_{S_{i}}\right)\right|} \\
& \underline{\operatorname{Sim}}\left(S_{i}, Q\right)=\frac{\left|\underline{N_{B}}\left(X_{Q}\right) \bigcap \underline{N_{B}}\left(X_{S_{i}}\right)\right|}{\left|\underline{N_{B}}\left(X_{Q}\right) \bigcup \underline{N_{B}}\left(X_{S_{i}}\right)\right|}
\end{aligned}
$$

where $\overline{N_{B}}(\square)$ and $\frac{N_{B}(\square)}{}$ are the neighborhood upper and lower approximation based on the reduced features $B$, respectively. ${ }^{X_{Q}}$ denotes the subset of the samples set $U$, which includes the query image $Q$. Similarly ${ }_{S_{i}}$ denotes the subset of the samples set $U$, which 
includes $S_{i} . \alpha$ is the coefficient to adjust the contribution of the neighborhood upper and lower approximation, and the range of the value of $\alpha$ is $[0,1]$.

In the image database $G, S_{i}$ and $S_{j}$ are the arbitrary two images belong to $G$. Then the similarity ratio between $S_{i}$ and $S_{j}$ conforms to the following properties:

Property 7: $\operatorname{Sim}\left(g_{i}, g_{j}\right)=\operatorname{Sim}\left(g_{j}, g_{i}\right)$.

Property 8: $\operatorname{Sim}\left(g_{i}, g_{i}\right)=1$.

Property 9: $0 \leq \operatorname{Sim}\left(g_{i}, g_{j}\right) \leq 1$.

According to Definition 5, the complete description of similarity measurement is given in Algorithm 2.

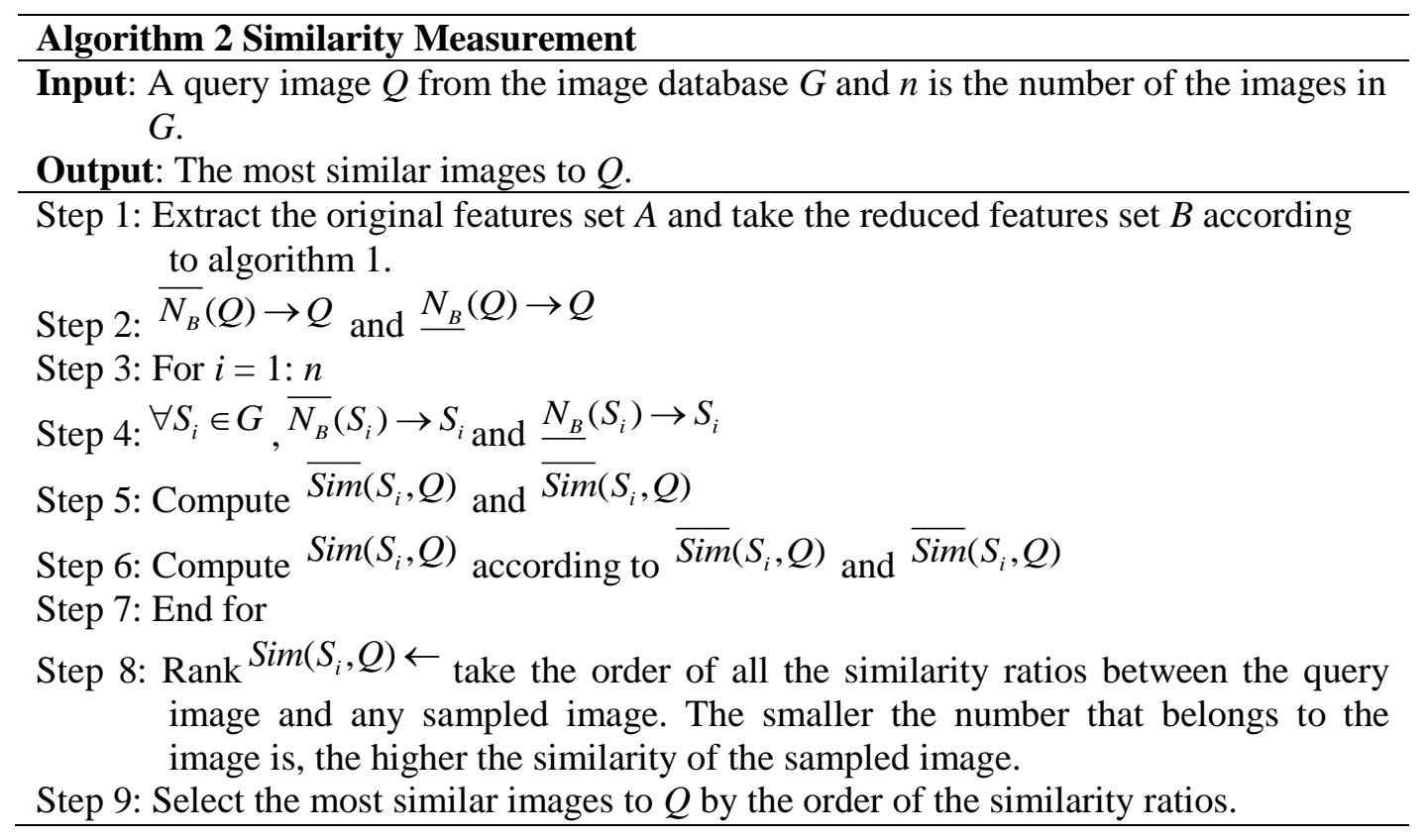

\section{Experimental Results}

In this section we provide the details of our experiments which evaluate the performance of the proposed methods. The color image retrieval systems have been implemented in MATLAB 2012 on a Core i5 (8 GHz) PC.

\subsection{Data Sets}

To check the retrieval efficiency of proposed method, we have tested the performance with a general purpose image database that consists of 10,000 images of 100 categories from the Corel Image Gallery [18]. Corel images have been widely used by the image processing and CBIR research communities. They cover a variety of topics, such as "bus", "food", "sunset", "elephant", "flower", etc; each image is scaled to a 240*240 pixel size.

We extract a set of image features A of each image by four kinds of features, including Fuzzy linking color histogram, location histogram, SIFT histogram and DOG texture histogram.

We compute a 64-dimensional Fuzzy linking color histogram created by a fuzzy inference system, and a 36-dimensional location histogram by quantizing the image locations into a $6 \times 6$ grid. We extract 256-dimension SIFT histogram of four scales using the LLC algorithm. In additional, the 30-dimensional texture histogram is extracted by the 
oriented DOG filters. We thus obtain a 386-dimensional $(64+36+256+30)$ feature vector $A_{i}$ for each image in the database $S_{i}$.

\subsection{Performance Measures}

The most common evaluation measures used in the image retrieval are precision and recall, which is showed as the following equations:

Precision $=$ Similar retrieved $/$ Total retrieved

Recall $=$ Similar retrieved $/$ Similar exist

Firstly, we empirically study the impact of parameter $\alpha$ based on the image database. Figure 1 and Figure 2 show that the values of the average precision and the average recall change along with the change of parameter $\alpha$. Obviously, the performance is relatively stable when the parameter $\alpha$ change to a certain range, such as $0.2<\alpha<0.5$.

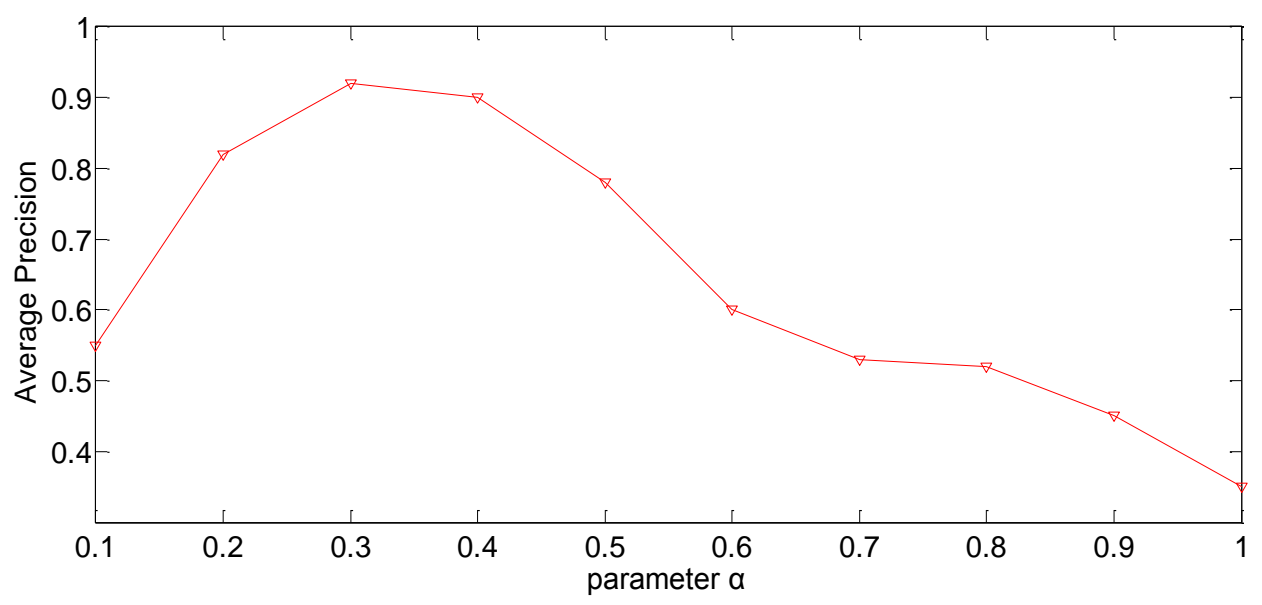

Figure 1. Precision Varies with $a$

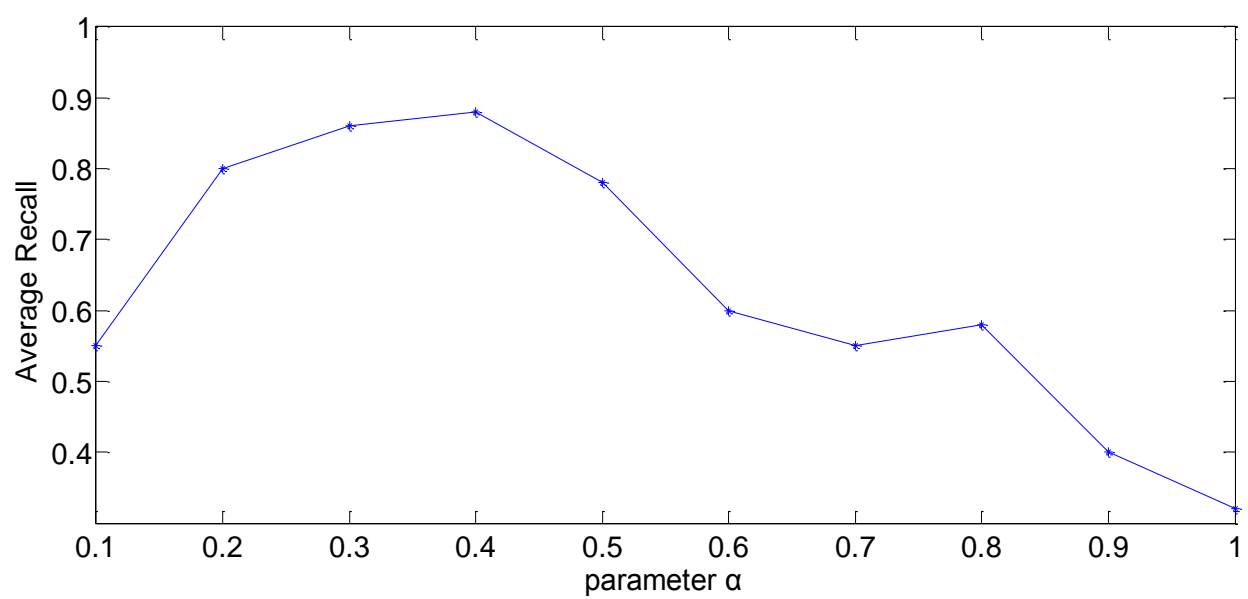

Figure 2. Recall Varies with $\alpha$

In order to intuitively illustrate the performance of the proposed algorithm, we stochastically the images as the query images from the Corel image database to achieve the retrieval results. Figure 3 shows the image retrieval results using the conventional similarity measurement and the proposed approach respectively. The image at the top of left-hand corner is the query image; the 10 images are the retrieval results that are the most 10 first similar to the query image. For the proposed approach, we set the weighting parameter in the experiment, i.e. $\alpha=0.35$. 

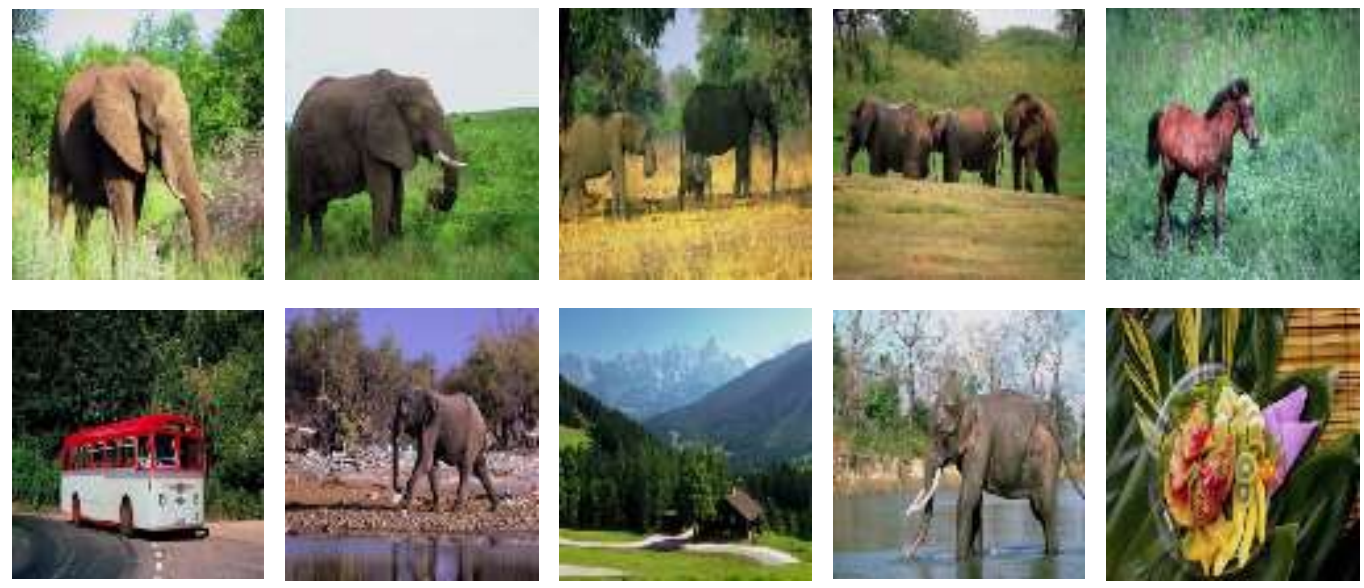

(a) The Conventional Similarity Measurement
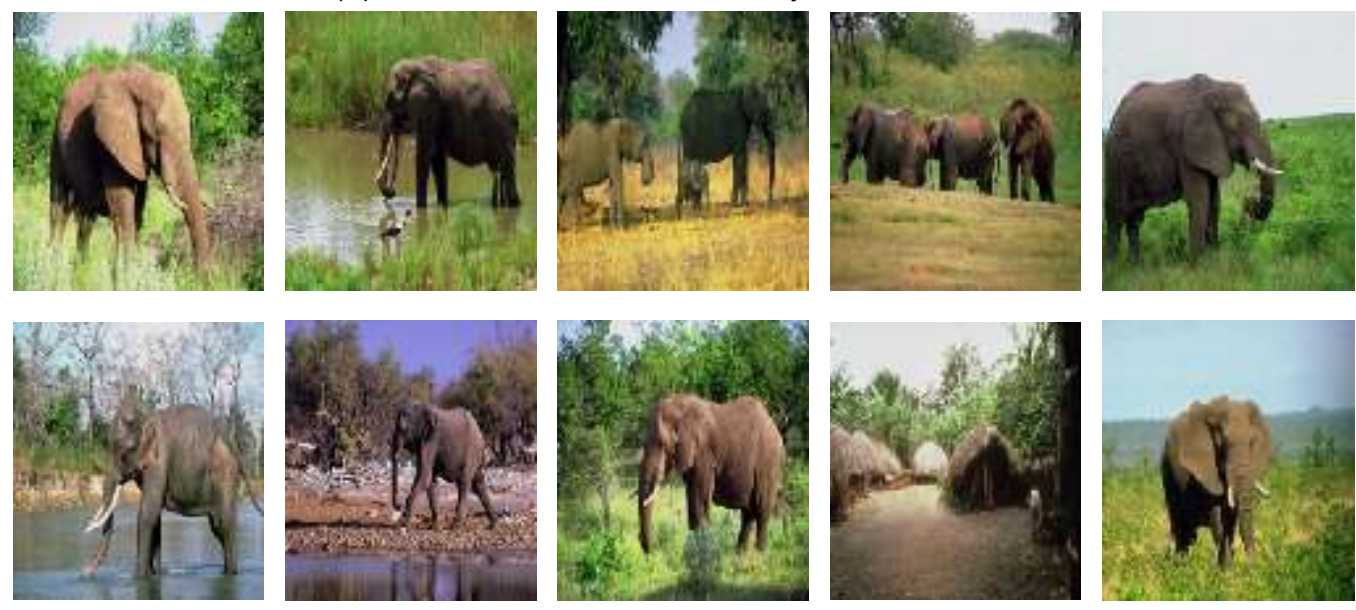

(b) The Proposed Algorithm

Figure 3. The Image Retrieval Results (Elephant)

In order to further confirm the validity of the proposed algorithm, we randomly selected 10 kinds to form the test image database, which contains total 1000 images. The 10 class includes bus, elephant, flower, building, horse, people, beach, scenery, bird and food. Each kind is extracted 10 images, and each time returns the first 20 most similar images as retrieval results. To each kind of images, the average precision, the average recall, and the average retrieval time of 10 times query results are calculated. These values are taken as the retrieval performance standard of the algorithm. The proposed method is compared with the other two methods. Method 1 [19] created the histogram of HIS color space that approximates the way of humans perceive color. The histogram consists of 20 bins, 16 for hue and 4 for intensity by selecting only $\mathrm{H}$ and I elements. Method 2 [20] adopted color histogram, texture and moment invariants to generate the image retrieval method so that the produced results which are highly relevant to the content of the query image. The comparisons of three methods are shown in Figures 4-6. 


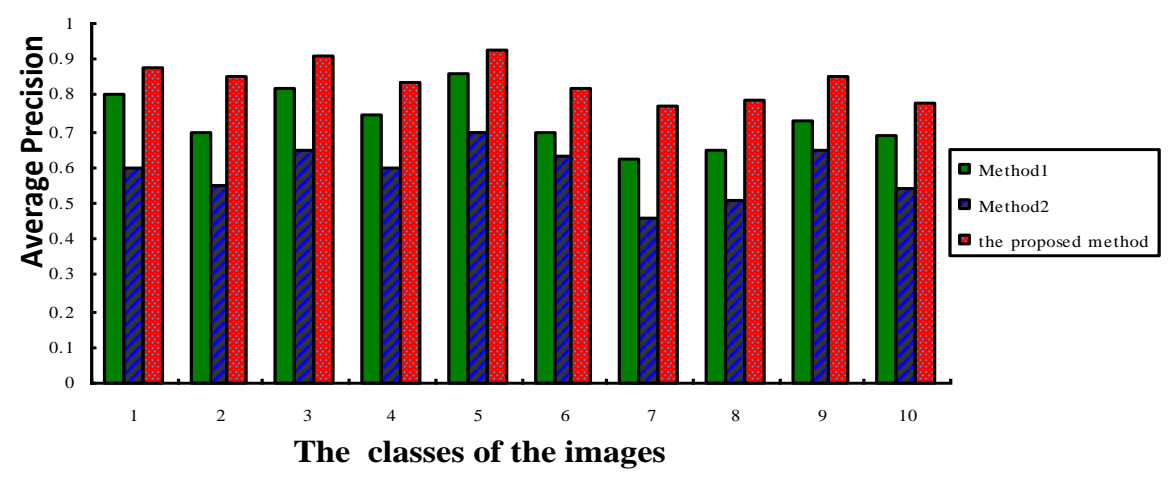

Figure 4. The Average Precision

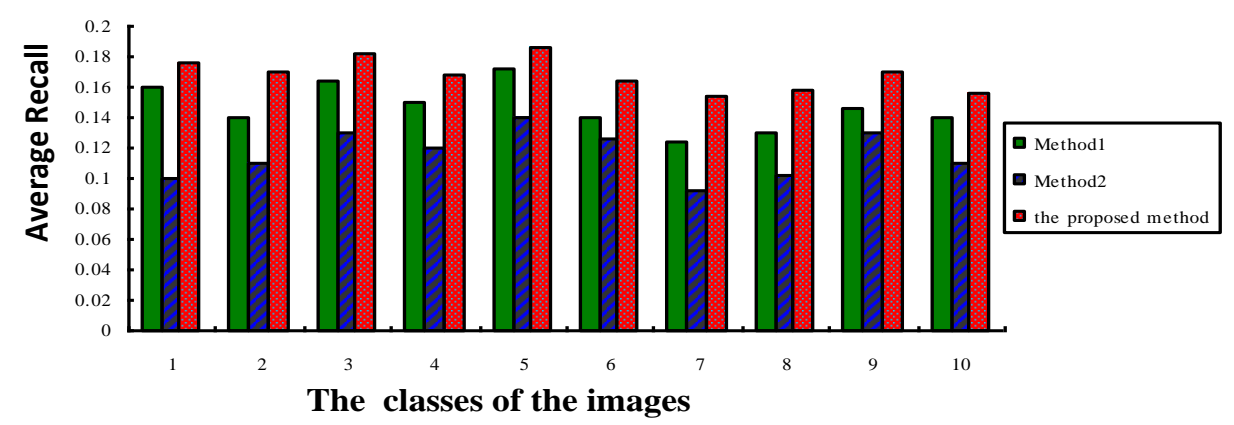

Figure 5. The Average Recall

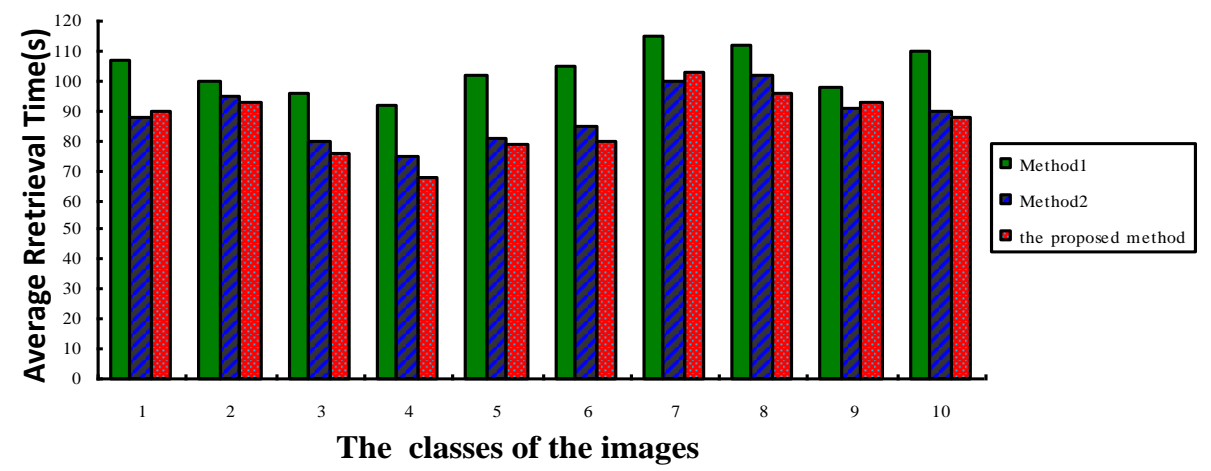

Figure 6.The Average Retrieval Time

Experimental results show that the proposed method performs best on more evaluation measures than the other methods. The reason for its good performance is that by introducing the upper and lower approximation of neighborhood rough set to find minimal reductions that is the optimal subset of the image features. It can increase the computation efficiency of the image retrieval. Furthermore, the similarity measurement through the neighborhood approximation concepts takes advantage of the features information in order to improve the classification capability of the image retrieval. So the proposed image retrieval method is more accurate and efficient in retrieving the interested images. 


\section{Conclusion}

The paper presented a method for using neighborhood rough set to achieve the reliable similar images to the query image. By introducing the upper approximation and lower approximation of neighborhood rough set, the proposed retrieval algorithm obtains better predictive performance than any other method while maintaining low retrieval time. Our future work is to research the more effective measurement of the similarity among the images.

\section{Acknowledgements}

This work is supported by the Education Innovation Foundation for Graduate Students of Shanxi Province (2015BY23).

\section{References}

[1] L. Zhuo, B. Cheng and J. Zhang, "A comparative study of dimensionality reduction methods for largescale image retrieval”, Neurocomputing, vol. 141, (2014), pp. 202-210.

[2] M. S. Lotfabadi, M. F. Shiratuddin and K. W. Wong, "Utilizing Fuzzy Rough Set Based on Mutual Information Decreasing Method for Feature Reduction in an Image Retrieval System", Springer International Publishing, (2015), pp. 177-184.

[3] J. J. Alpigini, J. F. Peters, J. Skowron and N. Zhong, "Rough sets and current trends in computing", Proceedings of the 3rd International Conference, USA, (2002).

[4] Z. Pawlak, "Rough sets", International Journal of Computer \& Information Sciences, vol. 11, no. 5, (1982), pp. 341-356.

[5] J. Li and N. Cercone, "A rough set based model to rank the importance of association rules", Rough Sets, Fuzzy Sets, Data Mining, and Granular Computing, Springer Berlin Heidelberg, (2005), pp. 109118.

[6] K. Thangavel and A. Pethalakshmi, "Dimensionality reduction based on rough set theory: A review", Applied Soft Computing, vol. 9, no. 1, (2009), pp. 1-12.

[7] J. Zaluski, R. Szoszkiewicz, J. Krysinski and J. Stefanowski, "Rough set theory and decision rules in data analysis of breast cancer patients", Transactions on Rough Sets 1, LNCS 3100, (2004), pp. 375-391.

[8] L. P. Khoo, S. B. Tor and L. Y. Zhai, "A rough set-based approach for classification and rule induction", International Journal of Advanced Manufacturing Technology, vol. 15, (1999), pp. 438-444.

[9] D. Chen, E. C. C Tsang, D. S. Yeung and X. Wang, "The parameterization reduction of soft sets and its applications", Computers \& Mathematics with Applications, vol. 49, no. 5, (2005), pp. 757-763.

[10] K. Thangavel, A. Pethalakshmi and P. Jaganathan, "A comparative analysis of feature selection algorithms based on rough set theory", International Journal of Soft Computing, vol. 1, no. 4, (2006), pp. 288-294.

[11] F. Questier, I. A. Rollier, B. Walczak and D. L. Massart, "Application of rough set theory to feature selection for unsupervised clustering", Chemometrics and Intelligent Laboratory Systems, vol. 63, no. 2, (2002), pp. 155-167.

[12] Y. Y. Yao, "Relational interpretations of neighborhood operators and rough set approximation operators", Information sciences, vol. 111, no. 1, (1998), pp. 239-259.

[13] Q. Hu, J. Liu and D. Yu, "Mixed feature selection based on granulation and approximation", Knowledge-Based Systems, vol. 21, no. 4, (2008), pp. 294-304.

[14] Y. Yu, W. Pedrycz and D. Miao, "Neighborhood rough sets based multi-label classification for automatic image annotation", International Journal of Approximate Reasoning, vol. 54, no. 9, (2013), pp. 1373-1387.

[15] P. Wu, S. C.-H. Hoi, P. Zhao, and Y. He. "Mining social images with distance metric learning for automated image tagging", Proceedings of the 4th ACM International Conference on Web Search and Data Mining, (2011), pp. 197-206.

[16] J. Yu, D. Tao, J. Li and J. Cheng. "Semantic preserving distance metric learning and applications", Information Sciences, vol. 281, (2014), pp. 674-686.

[17] H. Xia, P. Wu and S. C. H. Hoi, "Online multi-modal distance learning for scalable multimedia retrieval", Proceedings of the sixth ACM international conference on Web search and data mining. ACM, (2013), pp. 455-464.

[18] M. A. Stricker and A. Dimai. "Color indexing with weak spatial constraints", Proceedings of International Society for Optics and Photonics Electronic Imaging: Science \& Technology, (1996), pp. 29-40.

[19] M. Tico, T. Haverinen and P. Kuosmanen, "A method of color histogram creation for image retrieval", Nordic Signal Processing Symposium, (2000), pp. 157-160. 
[20] K. Iqbal, M. O. Odetayo and A. James, "Content-based image retrieval approach for biometric security using colour, texture and shape features controlled by fuzzy heuristics", Journal of Computer and System Sciences, vol. 78, no. 4, (2012), pp. 1258-1277. 
International Journal of Multimedia and Ubiquitous Engineering Vol.12, No.1 (2017) 